\title{
Autologous transplantation of genetically modified iris pigment epithelial cells: A promising concept for the treatment of age-related macular degeneration and other disorders of the eye
}

\author{
Irina Semkova*, Florian Kreppel*, Gerhard Welsandt ${ }^{\dagger}$, Thomas Luther ${ }^{\dagger}$, Jolanta Kozlowski ${ }^{\ddagger}$, Hanna Janicki ${ }^{\dagger}$, \\ Stefan Kochanek*§, and Ulrich Schraermeyer ${ }^{\dagger \neq}$
}

*Center for Molecular Medicine Cologne (ZMMK), ${ }^{\dagger}$ Center of Ophthalmology, Department of Retinal Surgery, ${ }^{\ddagger}$ Institute for Anatomy, University of Cologne, Kerpener Strasse 34, D-50931 Cologne, Germany

Communicated by C. Thomas Caskey, Cogene Biotech Ventures, Ltd., Houston, TX, August 13, 2002 (received for review March 8, 2002)

\begin{abstract}
Age-related macular degeneration (ARMD) is the leading cause for visual impairment and blindness in the elder population. Laser photocoagulation, photodynamic therapy and excision of neovascular membranes have met with limited success. Submacular transplantation of autologous iris pigment epithelial (IPE) cells has been proposed to replace the damaged retinal pigment epithelium following surgical removal of the membranes. We tested our hypothesis that the subretinal transplantation of genetically modified autologous IPE cells expressing biological therapeutics might be a promising strategy for the treatment of ARMD and other retinal disorders. Pigment epithelium-derived factor (PEDF) has strong antiangiogenic and neuroprotective activities in the eye. Subretinal transplantation of PEDF expressing IPE cells inhibited pathological choroidal neovascularization in rat models of laserinduced rupture of Bruch's membrane and of oxygen induced ischemic retinopathy. PEDF expressing IPE transplants also increased the survival and preserved rhodopsin expression of photoreceptor cells in the RCS rat, a model of retinal degeneration. These findings suggest a promising concept for the treatment of ARMD and other retinal disorders.
\end{abstract}

$\mathrm{n}$ the western world in individuals over 60 years of age, age-related macular degeneration (ARMD) is the primary cause for visual impairment and blindness. Pathological changes in the retinal pigment epithelium (RPE) and in the choriocapillaris are associated with the progressive degeneration of macular photoreceptor cells. Vision loss in patients with the exudative form of ARMD (wet ARMD) is caused by the formation of subretinal fibrovascular membranes during a late stage of the disease. When this occurs it sometimes can be ablated by laser photocoagulation or photodynamic therapy, but neither treatment addresses underlying stimuli for blood vessel growth. Therefore, recurrent disease is a problem of significant clinical relevance. Surgical excision of submacular neovascular membranes leads to the additional damage of the RPE (1), a cell type that is known to play a central role in the homeostasis of the retina having phagocytic, metabolic, neurotrophic, antioxidative, and antiangiogenic functions. The most frequent form of ARMD (dry ARMD) is characterized by primary degeneration and atrophy of the RPE layer. Subretinal transplantation of autologous (from the same patient) iris pigment epithelial (IPE) cells has been a recent experimental treatment strategy to functionally replace the lost or damaged RPE in the macular area $(2,3)$. The IPE is anatomically continuous with the RPE and has the same embryonic origin. In vitro, IPE cells share functional properties with RPE cells such as phagocytosis $(4,5)$, degradation of rod outer segments (ROS) (6), and synthesis of trophic factors (7). However, autologous transplantation of IPE cells has not resulted in a prolonged improvement of the vision of ARMD patients, potentially because of the lack of expression of one or several (so far unknown) factors that are an important part of RPE function. A promising strategy for the treatment of ARMD could be the transplantation of autologous IPE cells following modification with genes coding for antiangiogenic and/or neuroprotective proteins. Pigment epithelium-derived factor (PEDF) is a candidate therapeutic for ARMD because it has both antiangiogenic $(8,9)$ and neurotrophic $(10,11)$ activities in the eye. Naturally, PEDF is produced by RPE cells (12), human ciliary epithelium (13), and photoreceptors (14). Its endogenous synthesis is down-regulated by hypoxia (8) and senescence $(12,15)$. Therefore, increasing PEDF levels in the subretinal space might be an interesting strategy to control pathological angiogenesis in the retina. In recent experiments, overexpression of PEDF by $\Delta \mathrm{E} 1$ and $\Delta \mathrm{E} 1 / \Delta \mathrm{E} 4$-adenovirus vector-mediated gene transfer to the RPE inhibited retinal and choroidal neovascularization (16). In this study, earlier generation adenovirus vectors were used that, because of toxicity and immunogenicity, are unlikely to be suitable for clinical applications requiring long-term gene expression. Transduction of RPE and photoreceptor cells with a PEDF-expressing AAV vector also resulted in inhibition of choroidal neovascularization (CNV) (17). We reported long-term transgene expression following high-capacity adenovirus (HC-Ad) delivery of reporter gene to the RPE (18). Advantages of HC-Ad vectors [also called helper-dependent (HD) or "gutless" adenoviral vectors] are low toxicity and low immunogenicity, their potential to allow stable transgene expression following gene transfer, and their ability to efficiently deliver foreign DNAs with sizes of up to $36 \mathrm{~kb}$ into target cells (19). In patients with ARMD, the RPE degenerated and/or is not fully competent functionally. Therefore, autologous transplantation of genetically modified IPE cells to both replace the biological functions of the degenerated RPE and to add a therapeutic principle could be advantagous over a direct in situ gene transfer approach. Here, we generated an HC-Ad vector expressing human PEDF, transduced cultured IPE cells, and, after subretinal transplantation of these modified cells, tested their potential to inhibit neovascularization and to improve the survival of photoreceptors in relevant animal models of neoangiogenesis and retinal degeneration.

\section{Methods}

HC-Ad Vectors. The HC-Ad vectors were produced as described (20). The vector HC-Ad.PEDF was constructed to express the

Abbreviations: PEDF, pigment epithelium-derived factor; ARMD, age-related macular degeneration; IPE, iris pigment epithelium; RPE, retinal pigment epithelium; P, postnatal day; EGFP, enhanced GFP; HC-Ad, high-capacity adenovirus vector; hCEC, human choroidal endothelial cell; moi, multiplicity of infection; ROS, rod outer segments; CM, conditioned media; CNV, choroidal neovascularization.

§To whom reprint requests should be addressed. E-mail: Stefan.Kochanek@medizin. uni-koeln.de. 
human PEDF carrying a C-terminal 6-His tag (see Fig. 5, which is published as supporting information on the PNAS web site, www.pnag.org). The vector HC-Ad.FK7 expressing enhanced GFP (EGFP) has been described (21).

IPE, RPE, and Human Choroidal Endothelial Cells (hCECs). IPE (22) and RPE (23) cell cultures were prepared from Long Evans rats. Early passage cultures of hCECs (positive for von Willebrand factor) were obtained from donor eyes (24).

PEDF Detection. IPE cells $\left(1 \times 10^{6}\right)$ were transduced with 50 multiplicity of infection (moi) of HC-Ad.PEDF for $24 \mathrm{~h}$. Supernatants were collected 48 and $72 \mathrm{~h}$ after medium change. Secreted PEDF was detected by ELISA with mouse penta-His $(5 \mu \mathrm{g} / \mathrm{ml}$; Qiagen) and PEDF antibodies $(1 \mu \mathrm{g} / \mathrm{ml}$; Chemicon). Endogenous PEDF was determined from nontransduced IPE and RPE cells.

Effects of IPE-Produced PEDF on hCEC Function. IPE cells were transduced with 50 moi of HC-Ad.PEDF or 50 moi of HCAd.FK7. Undiluted, 1:10 diluted, or 1:100 diluted supernatants (CM, conditioned media) collected $72 \mathrm{~h}$ after medium change were added to hCECs.

To analyze proliferation, $1 \times 10^{3}$ hCECs per well seeded in 96-well plates were exposed to CM with or without $50 \mathrm{ng} / \mathrm{ml}$ VEGF (Sigma) and with or without $1 \mu \mathrm{g} / \mathrm{ml}$ anti-PEDF antibody. Five days later, cellular proliferation was determined using the WST-1 proliferation assay (Roche).

To analyze migration, $5 \times 10^{3}$ hCECs per insert were incubate in modified Boyden chambers (ref. 25; FluoroBlock inserts, Becton Dickinson) in CM with or without VEGF $(50 \mathrm{ng} / \mathrm{ml})$ for $8 \mathrm{~h}$ at $37^{\circ} \mathrm{C}$. Migrated cells were visualized by the nuclear fluorescence dye [4',6-diamidino-2-phenylindole (DAPI), Alexis, Grünberg, Germany] and counted in three random fields per membrane.

To determine the formation of capillary-like tubes in response to PEDF, $1 \times 10^{4} \mathrm{hCECs}$ per well were seeded in 96-well plates coated with VEGF-containing ECM gel (Chemicon) for $24 \mathrm{~h}$ in $\mathrm{CM}$.

Preparation of IPE Cells for Transplantation. IPE cells were transduced with 50 moi of HC-Ad.FK7 or were cotransduced with 50 moi of HC-Ad.PEDF and HC-Ad.FK7. Before transplantation the medium was changed, the cells were washed twice with PBS, and suspensions of $5 \times 10^{4}$ cells per $\mu$ l were transplanted $(1 \mu \mathrm{l}$ per eye).

Subretinal Transplantation and Fluorescence Imaging. EGFP fluorescence in Wistar rats was monitored 7 days, $1,2,3$, and 4 months after subretinal injection (26) of HC-Ad.FK7transduced IPE cells by Scanning Laser Ophthalmoscopy (SLO, Rodenstock, Germany) and Fundus Ophthalmoscopy (Stereomicroscope, SV 11, Zeiss), and on RPE-choroidal flatmounts (27) by fluorescence microscopy (Axioplan, Zeiss).

Electron Microscopy. Four months after injection, the areas of HC-Ad.FK7-transduced IPE transplants were subjected to electron microscopy (26).

Immunodetection of PEDF Expressed from IPE Cells. PEDF expression in IPE transplants was visualized in $4 \%$ paraformaldehydefixed RPE-choroidal flat-mounts and in paraffin sections by using penta-His antibody $(5 \mu \mathrm{g} / \mathrm{ml})$ and Cy3- or peroxidaseconjugated secondary antibodies (Amersham Pharmacia).

Oxygen-Induced Retinopathy. A model of ischemia-induced retinopathy (28) was set up in Wistar rats. Animals in the normoxia group were maintained in room air. Rats in the hyperoxia groups were exposed to $75 \%$ oxygen from postnatal day 7 (P7) to P12, then removed to room air and immediately transplanted subretinal with $(i)$ nontransduced IPE cells; (ii) IPE cells transduced with HC-Ad.FK7; and (iii) IPE cells cotransduced with HC-Ad.PEDF and HC-Ad.FK7. At P 22, the animals were anesthetized and perfused with $50 \mathrm{mg} / \mathrm{ml}$ fluorescein isothiocyanate-dextran (Sigma) as described (28). To quantify neovascularization, the length of newly formed tortuous blood vessels with diameters larger than $25 \mu \mathrm{m}$ on the inner surface of peripheral retina was determined as described (29) by computerassisted morphometry (OPENLAB software, ImproVision, Inc., Lexington, KY). A second quantification of neovascularization was performed by evaluation the sizes of vascularized areas. The fluorescence images were evaluated by setting of threshold level of fluorescence, above which the superficial retinal vessels but not the vessels of retinal deep plexus were captured. Vascularized areas were determined by the ratio of the surface area of the vessels to the total area of the retina in this region. To confirm epiretinal localization of the superficial pathological vessels, $10-\mu \mathrm{m}$ serial frozen sections were prepared, cut through half of the eye.

Laser-Induced CNV. IPE cells were subretinally transplanted into 4-5-month-old Long Evans rats. Six days later laser photocoagulation $(100 \mu \mathrm{m}$ spot size, $0.1 \mathrm{sec}$ duration, $150 \mathrm{~mW})$ was performed using the blue-green setting of a coherent Novus 2000 argon laser (Coherent Radiation, Palo Alto, CA) close to the transplanted IPE cells (three burns per eye). Ten days later, CNV was evaluated on RPE-choroidal flat-mounts by labeling endothelial cells with a rat anti-mouse CD31 (PECAM-1) monoclonal antibody ( $1 \mu \mathrm{g} / \mathrm{ml}$; Becton Dickinson). The sizes of the areas occupied with endothelial cells were determined by computer-assisted morphometry. The burns were classified as follows: type I, $100 \%$ occupied; type II, completely free from endothelial cells; type III, both occupied and endothelial cellfree areas present.

IPE Transplantation in RCS Rats. Two months after transplantation of IPE cells into 20-day-old RCS rats (26) the animals were killed and $5-\mu \mathrm{m}$-thick paraffin sections were prepared cut through half of the eye. After H\&E staining, rescue effects were quantified by determining the number of rows and size of areas with preserved photoreceptor nuclei in all sections. Rhodopsin expression was detected with rhodopsin antibody $(5 \mu \mathrm{g} / \mathrm{ml}$; Leinco Technologies, St. Louis) and peroxidase-labeled secondary antibody. The sizes of areas with preserved rhodopsin-containing outer segments were determined using computer-assisted morphometry.

Statistics. All data are means \pm SEM from at least three experiments with three to six determinations. For multiple comparisons one-way analysis of variance (ANOVA) was used with subsequent post hoc analysis (Duncan test).

\section{Results}

Endogenous and HC-Ad Vector-Mediated Production of PEDF in Vitro. RPE cells secreted $96 \pm 9.5 \mathrm{ng} / \mathrm{ml}$ endogenous PEDF within $72 \mathrm{~h}(n=4)$, whereas endogenous PEDF was not detected in supernatants from IPE cells (detection limit $1.56 \mathrm{ng} / \mathrm{ml}$ ). Following transduction of IPE cells with HC-Ad.PEDF vector, IPE cells $\left(1 \times 10^{6}\right.$ cells per dish, $\left.n=4\right)$ secreted $250 \pm 38 \mathrm{ng} / \mathrm{ml}$ PEDF into the supernatant within $72 \mathrm{~h}$ (see Fig. 6, which is published as supporting information on the PNAS web site).

We investigated the functional activity of vector-produced PEDF. First, nondiluted and 1:10 diluted CM from HCAd.PEDF-transduced IPE cells reduced VEGF-stimulated proliferation of hCECs without having any effect on nonstimulated proliferation. PEDF antibody $(1 \mu \mathrm{g} / \mathrm{ml})$, present in the $\mathrm{CM}$, abolished the inhibitory effect (data not shown). CM from 
HC-Ad.FK7-transduced and nontransduced IPE cells did not influence both stimulated and nonstimulated proliferation of the cells. Second, CM from HC-Ad.PEDF-transduced IPE cells reduced the migration of hCECs toward angiogenic VEGF from $47.5 \pm 5.9$ to $14.3 \pm 5.7$ cells per membrane $(P<0.001)$. Exposure to CM from HC-Ad.FK7-transduced and nontransduced IPE cells did not influence hCECs migration (data not shown). Third, CM from HC-Ad.PEDF-infected IPE cells suppressed neovascular tube formation, whereas supernatants from HC-Ad.FK7-transduced and nontransduced IPE cells had no effect (data not shown).

Long-Term EGFP Expression After Subretinal Transplantation of HCAd.FK7-Transduced IPE Cells. Seven days after transplantation of HC-Ad.FK7-transduced IPE cells, areas of patchy and/or continuous fluorescence were observed by SLO at the site of injection. Three months later, areas of bright EGFP fluorescence were still present in the same eyes with comparable extension and intensity as at 1 and 2 month (data not shown). Three months after surgery, the expression of EGFP in transplanted IPE cells was also investigated by Fundus Ophthalmoscopy (Fig. $1 a$ and $b$ ). Fig. $1 a$ shows the localization of IPE transplants in which bright EGFP fluorescence signal is still present three months after transplantation (Fig. 1b). Four months after surgery, EGFP expressing IPE cells were found integrated into the host RPE layer as determined on RPE-choroidal flat-mounts (Fig. 1c). By electron microscopy, pigmented IPE cells formed a second layer on the RPE of the Wistar hosts (Fig. 1d). The ROS facing the transplants appeared morphologically intact (Fig. 1d). Most of the transplanted IPE cells formed microvillar processes that attached to the tip of the ROS (Fig. 1e).

Effect of HC-Ad.PEDF Vector-Transduced IPE Cells in a Model of Ischemic Retinopathy. At P22, on flat-mounts from hyperoxia controls, large peripheral areas with dilated radial vessels, microaneurysms, and hemorrhages, typical for retinal neovascularization, were observed (Fig. 2a). Typical signs of peripheral retinal neovascularization characterized by vascular tufts, dilated vessels with abnormal tortuous courses on the inner surface of the peripheral retina were evident [Fig. $2 b$ (higher magnification of $2 a$ )]. The epiretinal position of the newly formed pathological vessels was evident in cryosections (Fig. 2c). These were not present in retinas from normoxia controls (data not shown). IPE cell transplants were localized in the peripheral areas of flatmounts between the RPE layer and the neuronal retina. IPE cells were identified by EGFP expression (Fig. $2 d$ and $e$ ) and their preserved hexagonal shape (Fig. 2f). HC-Ad.PEDF-transduced IPE implants prevented the formation of pathological tortuous vessels in the areas of transplantation (Fig. $2 d$ and $e$ ). Immunofluorescence microscopy documented the expression of PEDF from genetically modified IPE transplants (Fig. $2 f$ ). IPE transplants were located in the periphery where typical pathological vessels are usually formed after exposure to hyperoxia in this model (26). To quantify the neovascularization the length of dilated tortuous vessels on the inner surface of the retina with diameters larger than $25 \mu \mathrm{m}$ were measured on retinal areas of $800,000 \mu \mathrm{m}^{2}$ per eye. Evaluated areas were localized up to 200 $\mu \mathrm{m}$ from IPE transplants or in corresponding peripheral regions of hyperoxia controls (Fig. $2 b$ and $e$ ). Similar as in normoxia controls, in the vicinity of PEDF-expressing IPE cells pathological epiretinal vessels were absent. Nontransduced IPE cell transplants did not influence pathological vascularization compared with hyperoxia controls (see Fig. 7, which is published as supporting information on the PNAS web site). Additionally to the vessel length, the sizes of vascularized areas (superficial vessels) were determined as an independent parameter of neovascularization. Close to PEDF expressing IPE cells the sizes of vascularized areas were reduced to $0.0153 \pm 0.0081 \mu \mathrm{m}^{2}$ blood
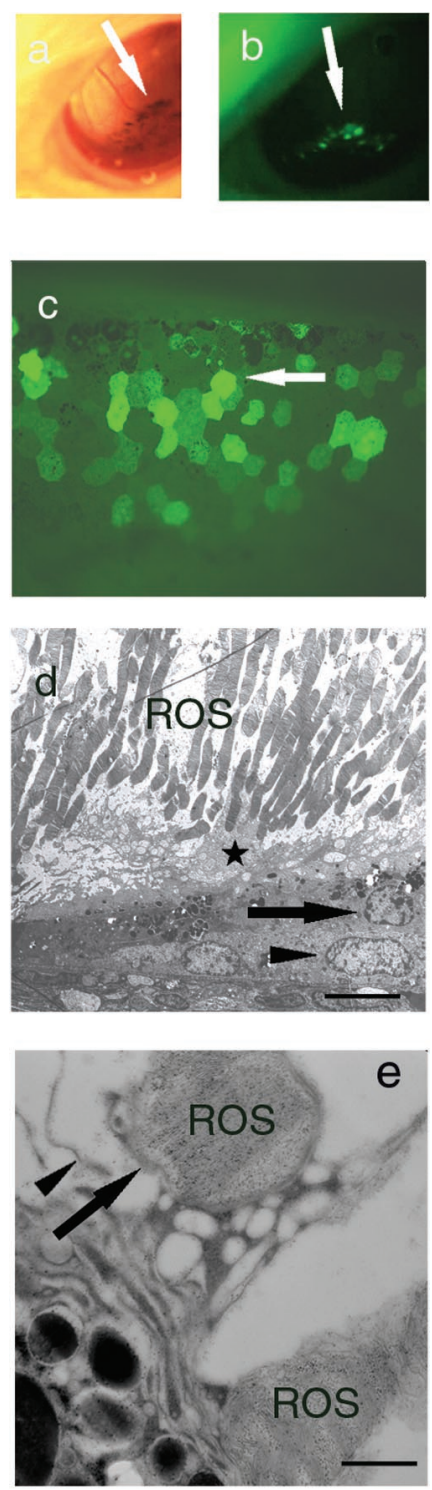

Fig. 1. Long-term expression of EGFP after subretinal transplantation of HC-Ad.FK7-transduced IPE cells. (a) Fundus Ophthalmoscopy image of IPE transplants (arrow) 3 months after transplantation. (b) Fundus Ophthalmoscopy fluorescence image of the same eye as in a showing bright EGFP fluorescence signal (arrow). (c) EGFP-expressing IPE cells on RPE-choroidal flatmount 4 months after transplantation. IPE cells preserved their hexagonal shape (arrow) and are well integrated on the host RPE layer. (d) Electron microscopy of the same IPE transplants as in c; IPE cells form a second layer on RPE. IPE and RPE cell nuclei are indicated by arrow and arrowhead, respectively. IPE cells with cytoplasmatic protrusions (star) are in close contact with ROS. At the ultrastructural level the photoreceptor-IPE interface is intact. (e) The IPE cells form microvillar processes (arrowhead). One of such processes (arrow) is closely attached to the tip of the rod outer segment. The tip of the rod at the lower right is also closely adherent with the surface of the IPE cell. [Scale bars: $30 \mu \mathrm{m}(c), 10 \mu \mathrm{m}(d)$, and $0.5 \mu \mathrm{m}(e)$.]

vessels per $\mu \mathrm{m}^{2}$ tissue in comparison to $0.0546 \pm 0.014 \mu \mathrm{m}^{2}$ blood vessels per $\mu \mathrm{m}^{2}$ tissue in retinal regions with transplanted nontransduced IPE cells.

Effects of HC-Ad.PEDF Vector-Transduced IPE Cells in a Model of Laser-Induced CNV. Laser scars were identified on bright field images by pigment clumping (Fig. $3 a$ ). Fig. $3 b$ is a fluorescence micrograph of a laser burn from nontransplanted laser controls shown in Fig. $3 a$ after labeling of the endothelial cells with the 

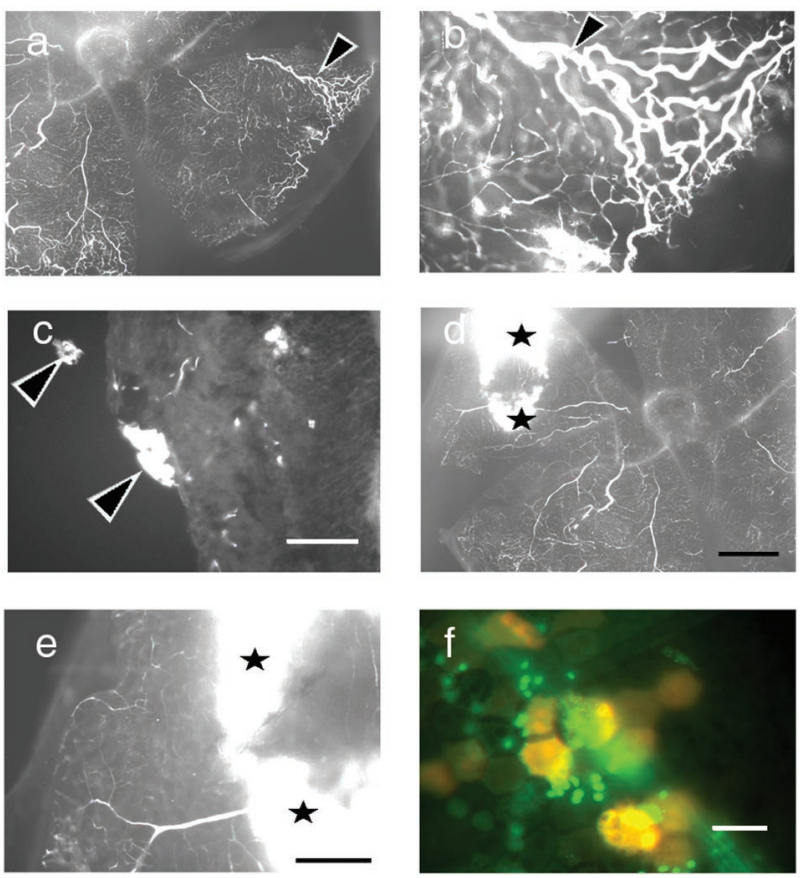

Fig. 2. Prevention of retinal neovascularization by IPE transplants expressing PEDF. ( $a$ and $b$ ) Fluorescence images of retinal flat-mount from nontransplanted hyperoxia controls. (a) Peripheral areas (arrowhead) with abnormal dilated vessels, microaneurysms, and hemorrhages. (b) Higher magnification of a. Newly formed vessels with abnormal tortuous course (arrowhead) on the inner surface of the retina. (c) Fluorescence image of cryosection cut through the eye from nontransplanted hyperoxia control. Arrowheads indicate epiretinal vessels at the surface of the retina (lower right) and vitreous (upper left). ( $d-f$ ) Fluorescence images of retinal flat-mount with EGFP expressing IPE transplants (cotransduced with HC-Ad.FK7 and HC-Ad.PEDF). (d) The bright fluorescent area (stars) corresponds to EGFP-marked IPE transplants localized between the RPE layer and the neural retina. (e) Higher magnification of the area close to HC-Ad.PEDF-transduced IPE transplants (stars) as in $d$. Note that retinal tissue is free from pathological tortuous vessels in the areas of transplantation. ( $f$ ) Expression of PEDF in EGFP marked IPE transplants. Fluorescence image after labeling of genetically induced PEDF with penta-His antibody and Cy3-conjugated secondary antibody. [Scale bars: $700 \mu \mathrm{m}$ ( $a$ and $d$ ), $240 \mu \mathrm{m}(b$ and $e), 100 \mu \mathrm{m}(c)$, and $16 \mu \mathrm{m}(f)$.]

PECAM-1 antibody. The burn (type I) is completely (100\%) occupied with newly formed endothelial cells. IPE transplants were localized surrounding the laser burns (Fig. $3 c, e$, and $f$ ). In Fig. 3c, EGFP-expressing IPE cells (cotransduced with HC.AdFK7 and HC-Ad.PEDF) close to three laser burns are shown. These IPE transplants strongly expressed PEDF as detected by staining with the penta-His antibody (Fig. $3 d$ ). Two of the laser burns surrounded from IPE cells in Fig. $3 c$ are shown in Fig. $3 e$ after immunolabeling with the endothelial cell marker. The presence of PEDF-expressing IPE transplants close to the site of damage prevented formation of new vessels within the laser burns (Fig. 3e). These laser burns were classified as type II burns, completely free from endothelial cells. Furthermore, PEDF-expressing IPE cells (about 100) surrounding the burn shown in Fig. $3 f$ reduced the area of neovascularization compared with nontransplanted laser controls (100\% occupation, Fig. $3 b)$. In this case, newly formed endothelial cells occupied $67 \%\left(47,400 \mu \mathrm{m}^{2}\right)$ of the whole area within the burn $(70,500$ $\mu \mathrm{m}^{2}$ ). Endothelial cells remained at the margin of the scar and did not proliferate or migrate into the center of the burn. The areas free of endothelial cells within the burn were localized close to transplanted IPE cells (Fig. $3 f$ ).

Table 1 (published as supporting information on PNAS web site) summarizes the classification of laser burns based on the
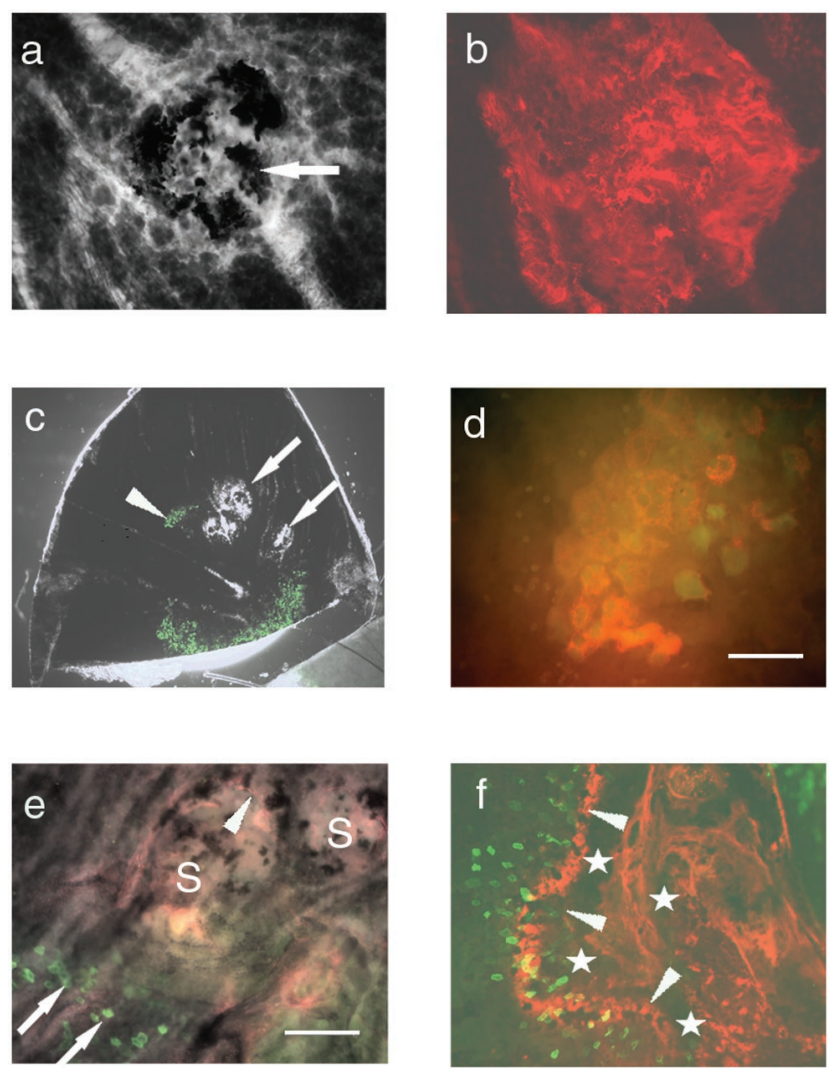

Fig. 3. Inhibition of laser-induced neovascularization by PEDF-expressing IPE transplants. (a) Bright field image of laser burn from nontransplanted control eye with typical pigment clumping (arrow). (b) Fluorescence micrograph from the same laser burn as in $A$ after labeling of endothelial cells with PECAM-1 antibody. The burn is completely occupied by newly formed vessels (type I). (c) Bright field and fluorescence image of EGFP marked IPE transplants (cotransduced with HC-Ad.FK7 and HC-Ad.PEDF; arrowhead) close to three laser burns (arrows). The IPE cells are localized surrounding the laser burns. (d) Fluorescence image of PEDF-expressing IPE cells (cotransduced with HC-Ad.FK7 and HC-Ad.PEDF). PEDF expressed from HC-Ad.PEDF was visualized by penta-His antibody and Cy3-labeled secondary antibody. (e) PEDF-expressing IPE transplants prevent neovascularization within the laser burns. Fluorescence image of the same IPE transplants (arrows) as in c and $d$ close to two laser burns shown in $c$ after labeling of endothelial cells with PECAM-1 antibody and Cy3 secondary antibody. Capillary-like tubes (arrowhead) are present at the margin of the scars (S) only. Laser burns surrounded from PEDF-expressing IPE cells are completely free from endothelial cells (type II). (f) IPE transplants reduce the vascularization within the laser burn (type III). Fluorescence image of laser burn surrounded from PEDF-expressing IPE cells after labeling of the endothelial cells. Endothelial cells are localized predominantly at the margin of the scar (arrowheads) and areas free of endothelial cells (stars) are present close to PEDF expressing IPE cells. The laser burn has a diameter of approximately $100 \mu \mathrm{m}(a-c)$. [Scale bars: $30 \mu \mathrm{m}(d)$ and $80 \mu \mathrm{m}(e$ and $f)$.]

size of CNV areas in the different experimental groups. Neither EGFP-expressing IPE nor nontransduced IPE transplants influenced laser-induced CNV formation.

Effects of HC-Ad.PEDF Vector-Transduced IPE Cells on Photoreceptor Survival in RCS Rats. Two months after surgery of RCS rats, only a few photoreceptor nuclei were present close to IPE cells that had been transduced with HC-Ad.FK7 alone (Fig. 4a). However, several rows of photoreceptor nuclei were preserved adjacent to the PEDF-transduced IPE transplants (Fig. 4b). In these areas PEDF was expressed from HC-Ad.PEDF-transduced IPE transplants (Fig. 4c). In these sections the number of photoreceptor rows was significantly higher $(4.4 \pm 0.68, P<0.05)$ compared with HC-Ad.FK7-transduced $(2.18 \pm 0.29)$ and nontransduced 

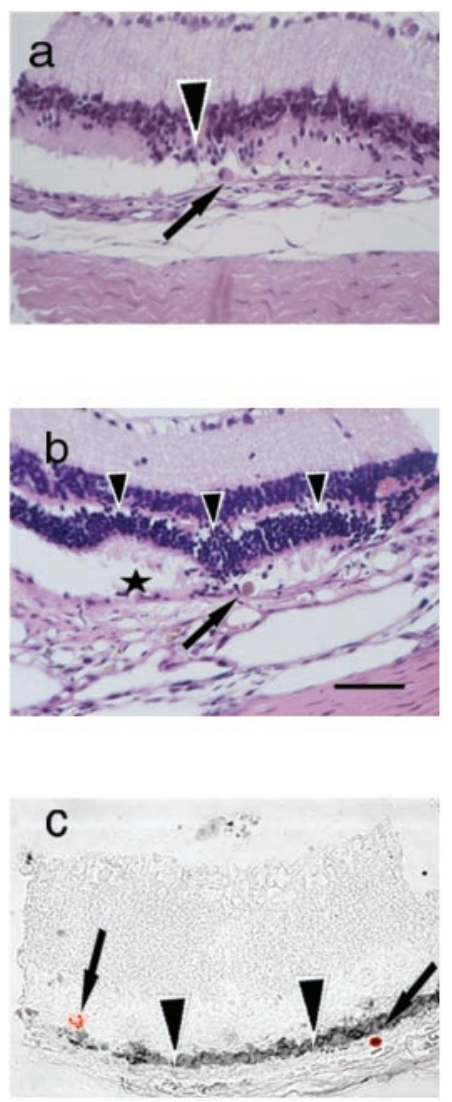

Fig. 4. PEDF-expressing IPE transplants increase survival and preserve the morphology of the photoreceptor cells in RCS rats 2 months after surgery. Bright field micrographs of H\&E-stained paraffin sections cut through half of the eye. (a) Subretinal transplanted IPE cells (arrow) transduced with HCAd.FK7. Only a few photoreceptor nuclei (arrowhead) are present close to transplants. (b) Many rows of photoreceptor nuclei (arrowheads) are preserved close to HC-Ad.PEDF-transduced IPE cells (arrow). ROS (star) are also present. (c) Preserved rhodopsin (arrowheads) in ROS of the photoreceptors on the same sections. PEDF-expressing IPE cells (arrow) are localized close to remnants of rhodopsin. [Scale bar: $100 \mu \mathrm{m}(a-c)$.]

IPE transplants $(2.2 \pm 0.55)$ (see Fig. 8, which is published as supporting information on PNAS web site). In addition, in sections with PEDF expressing cells $(n=9)$, the areas with preserved photoreceptor nuclei (more than five rows) had a length of $2.6 \pm 1.0 \mathrm{~mm}$. As shown in Fig. $4 c$, preserved rhodopsin was present in rod outer segments of the survived photoreceptors in the vicinity of PEDF-expressing cells. In the same sections a rhodopsin positive area of $53947 \pm 24656 \mu \mathrm{m}^{2}(n=5)$ was measured. In sections with HC-Ad.FK7-transduced or in nontransduced IPE transplants, rhodopsin staining was not detectable.

\section{Discussion}

The exudative form of ARMD is characterized by the formation of neovascular membranes protruding from the choroid through Bruch's membrane into the subretinal space. This process is mainly localized to the macular region and invariably leads to rapid and significant vision loss. During submacular surgery not only the membranes but also the RPE cell layer, which is known to play a central role in the homeostasis of the retina, is removed. In the dry form of ARMD, primary geographic atrophy of the RPE leads to the degeneration of macular photoreceptors

1. Castellarin, A. A., Nasir, M. A., Sugino, I. K. \& Zarbin, M. A. (1998) Br. J. Ophthalmol. 82, 480-487. resulting in vision loss. Submacular transplantation of autologous IPE cells has been proposed to replace damaged RPE. However, clinical studies have not unequivocally demonstrated clinical benefit $(2,3)$.

Here, we have suggested a treatment for ARMD based on transplantation of genetically modified autologous IPE cells. The most significant findings are summarized as follows: first, IPE cells were readily transduced with HC-Ad vectors. Second, genetically modified IPE cells formed a monolayer following transplantation and stably expressed an EGFP reporter for at least 4 months without adverse effects. Third, IPE cells, in contrast to RPE cells, were found to lack the expression of PEDF, a protein that is believed to play a key role in the homeostasis of the retina. Fourth, IPE cells secreted functionally active PEDF at high levels after HC-Ad-mediated gene transfer. Fifth, subretinal transplantation of PEDF-expressing IPE cells inhibited neovascularization in models of retinal neoangiogenesis, and prevented photoreceptor degeneration in a model of retinal degeneration. Any obvious adverse effects due to the procedure were not observed. However, further studies are needed to investigate the long-term consequences of this strategy and the optimal therapeutic concentration of PEDF that has to be achieved.

The angiogenesis-inhibiting effect of HC-Ad vector expressing PEDF was observed locally in the vicinity of the IPE transplants. This observation supports the concept that IPE cells modified to produce one or several secreted therapeutic molecules have a significant potential for the treatment of diseases in which a small tissue area such as the foveola $\left(0.1 \mathrm{~mm}^{2}\right)$, the most important for the vision macular region, has to be protected from insults including neovascularization. In the RCS rat, a model for retinal degeneration and ARMD, RPE cell transplantation has been shown to rescue photoreceptor cells from degeneration (30, 31). The rescue effect on photoreceptors by transplantation of HC-Ad.PEDF-modified IPE cells was considerably more prominent than following transplantation of nontransduced IPE cells in our earlier study (26). In patients with ARMD similar to that in the RCS rat, the RPE is functionally not fully competent. Thus, the transplantation of genetically modified autologous IPE cells to replace the RPE in the macula of ARMD patients might be advantageous over modifying a functionally incompetent RPE by direct gene transfer.

The extended capacity to carry foreign DNA of $36 \mathrm{~kb}$ size is a significant advantage of the vector type used in this study. The pathogenesis of ARMD is probably linked to genetic variables/ changes in several genes. Therefore, it is possible that the expression of more than one gene will be required to achieve optimal therapeutic effects, which would be difficult to achieve with most other available vector types. In addition, the inclusion of a gene switch system to control potent (such as antiangiogenic) biological activities has been demonstrated to be feasible with this vector type (32).

We conclude that the transplantation of autologous IPE cells after genetic modification to express PEDF and/or other biologicals has a significant clinical potential for the treatment of ARMD or other retinal disorders.

We are grateful to Noel Bouck (Northwestern University, Chicago) for providing the PEDF cDNA, and to Frank Graham (McMaster University, Hamilton, ON, Canada) for providing the helper virus AdLC8cluc. This work was supported by grants to S.K. and U.S. from the Federal Ministry of Education and Research (FKZ, 01KS9502) and the Center for Molecular Medicine Cologne, to U.S. from the Köln Fortune Program, the Retinovit Foundation, and the Propter Hominis Foundation, and to F.K. from the Boehringer Ingelheim Foundation.

2. Thumann, G., Aisenbrey, S., Schraermeyer, U., Lafaut, B., Esser, P., Walter, P. \& Bartz-Schmidt, K. U. (2000) Arch. Ophthalmol. 118, 1350-1355. 
3. Lappas, A., Weinberber, A. W., Foerster, K. T., Rezai, K. A. \& Kirchhof, B. (2000) Graefes Arch. Clin. Exp. Ophthalmol. 238, 631-641.

4. Rezai, K. A., Lappas, A., Farrokh-Siar, L., Kohen, L., Wiedemann, P. \& Heimann, K. (1997) Exp. Eye Res. 65, 23-29.

5. Flannery, J. G., O’Day, W., Pfeffer, B. A., Horwitz, J. \& Bok, D. (1990) Exp. Eye Res. 51, 717-728.

6. Schraermeyer, U., Enzmann, V., Kohen, L., Addicks, K., Wiedemann, P. \& Heimann, K. (1997) Exp. Eye Res. 65, 277-287.

7. Kociok, N., Heppekausen, H., Schraermeyer, U., Esser, P., Thumann, G., Grisanti, S. \& Heimann, K. (1998) Exp. Eye Res. 67, 237-250.

8. Dawson, D. W., Volpert, O. V., Gillis, P., Crawford, S. E., Xu, H., Benedict, W. \& Bouck, N. P. (1999) Science 285, 245-248.

9. Stellmach, V., Crawford, S. E., Zhou, W. \& Bouck, N. (2001) Proc. Natl. Acad. Sci. USA 98, 2593-2597.

10. Cao, W., Tombran-Tink, J., Chen, W., Mrazek, D., Elias, R. \& McGinnis, J. F. (1999) J. Neurosci. Res. 57, 789-800.

11. Cayouette, M., Smith, S. B., Becerra, S. P. \& Gravel, C. (1999) Neurobiol. Dis. 6, 523-532.

12. Tombran-Tink, J., Shivaram, S. M., Chader, G. J., Johnson, L. V. \& Bok, D. (1995) J. Neurosci. 15, 4992-5003.

13. Ortego, J., Escribano, J., Becerra, S. P. \& Coca-Prados, M. (1996) Invest. Ophthalmol. Visual Sci. 37, 2759-2767.

14. Karakousis, P. C., John, S. K., Behling, K. C., Surace, E. M., Smith, J. E., Hendrickson, A., Tang, W. X., Bennett, J. \& Milam, A. H. (2001) Mol. Vis. 7, 154-163.

15. Pignolo, R. J., Cristofalo, V. J. \& Rotenberg, M. O. (1993) J. Biol. Chem. 268, 8949-8957.

16. Mori, K., Duh, E., Gehlbach, P., Ando, A., Takahashi, K., Pearlman, J., Mori, K., Yang, H. S., Zack, D. J., Ettyreddy, D., et al. (2001) J. Cell. Physiol. 188, $253-263$.
17. Mori, K., Gehlbach, P., Yamamoto, S., Duh, E., Zack, D. J., Li, O., Berns, K. I. Raisler, B. J., Hauswirth, W. W. \& Campochiaro, P. A. (2002) Invest. Ophthalmol. Visual Sci. 43, 1994-2000.

18. Kreppel, F., Luther, T. T., Semkova, I., Schraermeyer, U. \& Kochanek, S. (2002) Invest. Ophthalmol. Visual Sci. 43, 1965-1970.

19. Kochanek, S. (1999) Hum. Gene Ther. 10, 2451-2459.

20. Parks, R. J., Chen, L., Anton, M., Sankar, U., Rudnicki, M. A. \& Graham, F. L. (1996) Proc. Natl. Acad. Sci. USA 93, 13565-13570.

21. Dinser, R., Kreppel, F., Zaucke, F., Blank, C., Paulsson, M., Kochanek, S. \& Maurer, P. (2001) Histochem. Cell Biol. 116, 69-77.

22. Hu, D. N., Ritch, R., McCormick, S. A. \& Pelton-Henrion, K. (1992) Invest. Ophthalmol. Visual Sci. 33, 2443-2453.

23. Chang, C. W., Defoe, D. M. \& Caldwell, R. B. (1997) Invest. Ophthalmol. Visual Sci. 38, 188-195.

24. Hoffmann, S., Spee, C., Murata, T., Cui, J. Z., Ryan, S. J. \& Hinton, D. R. (1998) Graefes Arch. Clin. Exp. Ophthalmol. 236, 779-784.

25. Tang, S., Gao, Y. \& Ware, J. A. (1999) J. Cell Biol. 147, 1073-1084.

26. Schraermeyer, U., Kociok, N. \& Heimann, K. (1999) Invest. Ophthalmol. Visual Sci. 40, 1545-1556.

27. McMenamin, P. G. (2000) Invest. Ophthalmol. Visual Sci. 41, 3043-3048.

28. Smith, L. E., Wesolowski, E., McLellan, A., Kostyk, S. K., D’Amato, R., Sullivan, R. \& D’Amore, P. A. (1994) Invest. Ophthalmol. Visual Sci. 35, 101-111.

29. Karnabatidis, D., Dimopoulos, J. C., Siablis, D., Papazafiropoulos, D., Kalogeropoulou, C. P. \& Nikiforidis, G. (2001) Acta Radiol. 42, 333-338

30. Li, L. X., Sheedlo, H. J. \& Turner, J. E. (1990) Prog. Brain Res. 82, 179-185.

31. Sheedlo, H. J., Li, L., Gaur, V. P., Young, R. W., Seaton, A. D., Stovall, S. V., Jaynes, C. D. \& Turner, J. E. (1992) Int. Rev. Cytol. 138, 1-49.

32. Burcin, M. M., Schiedner, G., Kochanek, S., Tsai, S. Y. \& O’Malley, B. W. (1999) Proc. Natl. Acad. Sci. USA 96, 355-360. 